\title{
Intraoperative radiotherapy in stage IIB adenocarcinoma of the uterine cervix: a retrospective study
}

This article was published in the following Dove Press journal:

OncoTargets and Therapy

19 November 2013

Number of times this article has been viewed

Ying Gao'

Zi Liu'

Fei Gao ${ }^{2}$

Xi Chen ${ }^{2}$

'Department of Radiotherapy Oncology, First Affiliated Hospital of Medical College of Xi'an Jiaotong University, Xi'an, People's Republic of China; ${ }^{2}$ Department of Surgery, Second Affiliated Hospital of Medica College of Xi'an Jiaotong University, Xi'an, People's Republic of China
Correspondence: Zi Liu

Department of Radiotherapy Oncology, First Affiliated Hospital of Medical College of Xi'an Jiaotong University, Xi'an 710061 , People's Republic of China

Tel +860298532 4019

Email liuzmail@।63.com
Objectives: Adenocarcinoma (AC) of the uterine cervix has a poor prognosis and is usually fatal. The aim of this study was to evaluate the clinical outcome and toxicity of intraoperative electron beam radiation therapy (IOERT) in advanced AC.

Methods: Twenty-seven women were treated with IOERT. Surgery consisted of gross negative surgical margins in 19 patients, positive surgical margins in two patients, and close surgical margins in six patients. Twenty-three patients received chemotherapy.

Results: The 5-year overall survival and disease-free survival rates were 21/27 (77.8\%) and 19/27 (70.4\%), respectively. A significantly better survival rate was found in patients with AC compared to patients with adenosquamous carcinoma. Tumor recurrence and metastasis were observed in seven patients $(25.9 \%)$. No central failure occurred whether or not the resection margins were positive. Two (7.4\%) patients developed peripheral neurotoxicity.

Conclusion: IOERT plus surgery may be feasible and effective with a low risk of toxicity, even in patients with positive resection margins.

Keywords: IOERT, recurrence, adenocarcinoma, cervical cancer

\section{Background}

Adenocarcinoma (AC) of the uterine cervix currently accounts for 5\%-20\% of all cervical malignancies, and appears to be increasing in prevalence, ${ }^{1}$ presumably due to its rise in incidence in young women. ${ }^{2}$ Moreover, it has been reported that these tumors tend to metastasize to the lymph nodes, and are less sensitive to radio- and chemotherapy than squamous cell carcinoma (SCC). ${ }^{3}$ Most of our knowledge about the treatment of cervical cancer comes from studies where the majority of patients had SCC; however, few studies have reported on patients who have AC of the cervix. ${ }^{4-6}$ The little that is known about this disease points to the fact that it has a poor prognosis and is usually fatal. Thus, improvement in the therapy of $\mathrm{AC}$ of the uterine cervix is urgently needed.

Although the National Comprehensive Cancer Network guidelines include consideration of both intraoperative electron beam radiation therapy (IOERT) at the time of surgical exploration and resection as treatment options for patients with locoregional disease relapse, data to support this recommendation are scarce. IOERT is an innovative boosting technique which delivers a single high-dose fraction of radiotherapy directly to the resection bed during surgery to irradiate selective anatomical areas that have been identified during the surgical procedure as high risk and/or residual disease sites, while at the same time avoiding surrounding dose-limiting structures. This allows noncancerous intra-abdominal organs to be protected from receiving 
full doses of irradiation, which ultimately decreases the incidence of severe enteritis and increases the local control rates. ${ }^{7}$ Studies of IOERT in multiple anatomical sites have produced valuable results in terms of locoregional control and toxicity. ${ }^{8,9}$ It has been applied with some success in gynecologic malignancies, including endometrioid, cervical, vaginal, and ovarian cancers. ${ }^{10-13}$ Since 1997, IOERT has been used in patients at our institution for the treatment of a variety of gynecologic malignancies, particularly when resection margins are in doubt. The current study evaluated survival and patterns of relapse and toxicity for patients undergoing IOERT for AC.

\section{Methods}

\section{Patients}

This study was a nonrandomized trial. Between January 1999 and January 2007, 202 women with cervical cancer were treated with IOERT at the First Affiliated Hospital of Medical College of Xi'an Jiaotong University. Among them, 27 women with AC were diagnosed with International Federation of Gynecology and Obstetrics (FIGO) stage IIB. Patients with prior postoperative adjuvant treatments, such as chemotherapy, were allowed to enter the study. The present study was approved by the ethics committee of the First Affiliated Hospital of Medical College of Xi'an Jiaotong University. All patients gave signed informed consent for IOERT. Inclusion criteria were: 1) histologically proven AC; 2) FIGO stage IIB; 3) age < 75 years; 4) no evidence of pelvic or para-aortic lymph node metastasis on a computed tomography (CT) scan; 5) no evidence of metastasis on chest $\mathrm{X}$-ray; 6) normal liver and kidney function; 7) normal blood count; and 8) hemoglobin $>9$ g/L. Patients with SCC or previous pelvic radiotherapy were excluded from this analysis. The following criteria were studied: initial FIGO stage, initial performance status, response to IOERT, time and sites of relapse, overall survival (OS), and disease-free survival (DFS). Disease progression was defined as: new lesions that were consistent with new sites of disease on imaging that included CT, magnetic resonance imaging, ultrasound, and/or plain X-ray; biopsy/histology of new lesions; and new signs on clinical exams or symptoms consistent with new sites of disease.

\section{Preoperative therapy}

Patients underwent weekly intracavitary brachytherapy (dose of 12-14 Gy to International Commission on Radiation Units and Measurements reference Point A in two insertions using ${ }^{192}$ Ir was delivered).

\section{Intraoperative electron beam radiation therapy}

After a break of 1 to 2 weeks, all 27 patients underwent a simple hysterectomy with dissection of a palpable pelvic lymph node or tumor resection. During the operation, whole pelvic radiotherapy of 18-20 Gy was delivered intraoperatively using $12 \mathrm{Mev}$ electron beam (Varian 1800, Varian, Palo Alto, CA, USA). All treatments were accomplished using a single IOERT field. The superior border of the field was at the bifurcation of common iliac vessels, while the inferior border covered $2 \mathrm{~cm}$ inferior to the operated vaginal vault, and laterally covered $1 \mathrm{~cm}$ beyond the lateral margin of external and common iliac vessels. The electron intraoperative applicator was $10-12 \mathrm{~cm}$ in diameter. The bladder, intestines, and sigmoid colon were shifted out of the radiation field, and the rectum was shielded with a $6 \mathrm{~mm}$ thick lead sheet. The portion of the obturator nerve in the pelvic region was partially shifted out of the radiation field. ${ }^{8}$

\section{Postoperative therapy}

After 2 weeks of IOERT, chemotherapy was implemented with cisplatin and 5-fluorouracil at our institution. Cisplatin was given at a dose of $40 \mathrm{mg} / \mathrm{m}^{2} /$ day for 3 days, and 5 -fluorouracil was given by continuous fusion at a dose of $500 \mathrm{mg} / \mathrm{m}^{2} /$ day from the first to fifth day. The cycles were repeated every 3 weeks. Seventeen patients received four to six courses of chemotherapy, 2 to 3 weeks after IOERT. Six patients were treated with two courses of chemotherapy; four patients refused chemotherapy (Table 1).

Table I Patient characteristics

\begin{tabular}{ll}
\hline Characteristics & N \\
\hline Median age (range) & 46.2 years (2I-74) \\
Histologic subtype & 16 \\
Adenocarcinoma & 10 \\
Adenosquamous carcinoma & 1 \\
Other subtypes of adenocarcinoma & \\
Treatment & 4 \\
EBRT + IOERT & 17 \\
EBRT + IOERT + CT (4-6 cycles) & 6 \\
EBRT + IOERT + CT (2 cycles) & \\
Lymph node status & 2 \\
Positive & 9 \\
Negative & 16 \\
Unknown & \\
Margins & 2 \\
Positive & 19 \\
Negative & 6 \\
Close
\end{tabular}

Abbreviations: $\mathrm{CT}$, computed tomography; EBRT, external beam radiation therapy; IOERT, intraoperative electron beam radiation therapy. 


\section{Evaluation of acute and late toxicities}

Acute and late toxicities were graded by the Common Terminology Criteria for Adverse Events version 3.0. ${ }^{14}$ Complications that occurred within 90 days from the start of primary treatment were considered acute complications, and those that occurred more than 90 days after the start of treatment were considered late complications.

\section{Follow-up}

After the completion of treatment, patients were followed-up at 3-month intervals for 1 year, 6-month intervals for 3 years, and annually thereafter.

\section{Statistics}

OS and DFS curves were calculated according to the KaplanMeier method. $P$-values less than 0.05 were considered statistically significant.

\section{Results}

\section{Patient characteristics}

The median age of the 27 patients with $\mathrm{AC}$ at the time of diagnosis was 46.2 years (range, 21-74 years). All patients with primary tumors were diagnosed with FIGO stage IIB. Sixteen patients had AC (59.3\%), ten (37.0\%) had adenosquamous carcinoma (ASC), and one (3.7\%) had clear cell carcinoma. Dissection of a palpable pelvic lymph node was performed for eleven patients (57.8\%), and two of these patients $(18.2 \%)$ had lymph node metastasis. Patient characteristics are outlined in Table 1. The final pathology review following surgery revealed grossly negative surgical margins in 19 patients, positive surgical margins in two patients, and close surgical margins (in which the resected tumor had been adherent to unresectable vascular structures) in six patients.

During the follow-up interval, six (22.2\%) patients died; five patients (18.5\%) died of tumor-related disease, and one patient died of causes unrelated to cancer. No central failure occurred. Tumor recurrence and metastasis were observed in seven patients (25.9\%). Of the entire group, only two patients (7.4\%) relapsed due to locoregional failure (outside the IOERT boost), and five patients (18.5\%) had distant metastasis alone (including two cases of relapse para-aortic nodal metastasis, one case of liver metastasis, one case of multiple metastasis, and one case of lung metastasis). Among them, isolated local recurrence was detected in one patient (3.7\%), and combined local failure and distant metastasis were detected in one patient (3.7\%). The mean time to recurrence was 12 months (range, 8-24 months).

\section{OS and DFS}

The mean follow-up time was 81 months (range, 11-121 months). The 5-year OS and DFS rates in all 27 patients were $77.8 \%(21 / 27)$ and 19/27 (70.4\%), respectively. In total, six patients died. Among the five patients who died of tumor-related disease, $17.6 \%(3 / 17)$ were patients who were treated with chemotherapy for four to six courses compared to $20 \%(2 / 10)$ of patients who had less than four courses of chemotherapy or patients without chemotherapy. The 5-year OS and DFS in patients who had four to six courses of chemotherapy were $76.4 \%(13 / 17)$ and $70.6 \%(12 / 17)$, respectively. A $P>0.05$ was found for patients with less than four courses of chemotherapy $(8 / 10)$, or patients without chemotherapy $(7 / 10)$, as shown in Figure 1A and B. However, a significantly better survival rate was found in patients with $\mathrm{AC}(\mathrm{OS}, 15 / 16$; DFS, 14/16) compared to patients with adenosquamous carcinoma (OS, 6/10; DFS, 5/10) $(P=0.034, P=0.031)$ (Figure 1C and D and Table 2). The rates of distant metastasis were similar between patients (4/16 and 2/10, respectively; $P=0.768$ ). Local relapse was detected in $7.4 \%(2 / 27)$ of patients and central control (within the IOERT field) was $100 \%$ whether or not the resection margins were positive. No local failures occurred in any of the eight patients who underwent subtotal resection.

\section{Toxicities}

Generally, IOERT was well tolerated. The only noted toxicities were those potentially related to the local effects from local treatments. Toxicity related to chemotherapy was not included. In addition, no radiation-induced nausea or vomiting was observed, and no delayed wound healing occurred. None of the patients suffered from enteritis during the entire follow-up period. The major complication observed was peripheral neuropathy, which two (7.4\%) patients developed after 8 months and 17 months. The incidence of developing neuropathy was present while the peripheral nerve was included in the IOERT field. In addition, one patient suffered from abdominal pain $(3.7 \%)$. Other toxicities related to surgery, such as urinary tract infection, developed in 3.7\% of the patients. All cases were manageable by conservative treatment.

\section{Discussion}

AC has been always managed in the same manner as SCC. However, the majority of studies showed that AC carries a worse prognosis than SCC, with $10 \%-20 \%$ differences in 5 -year OS rates. ${ }^{15}$ Baalbergen et a ${ }^{16}{ }^{16}$ demonstrated in a study 
A

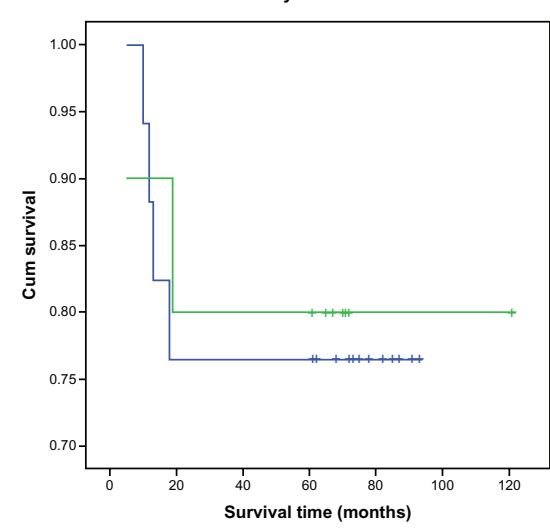

C

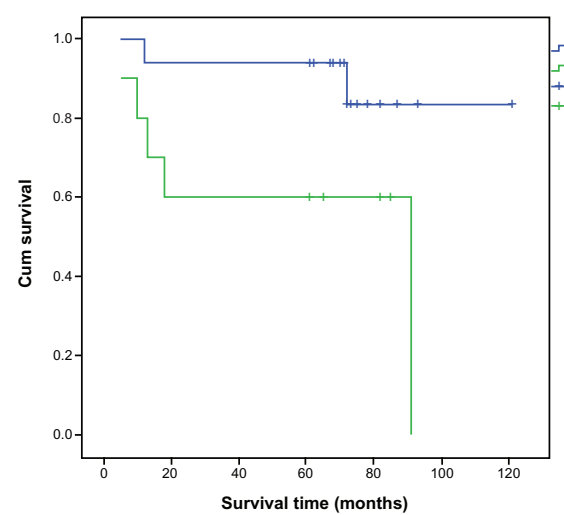

B
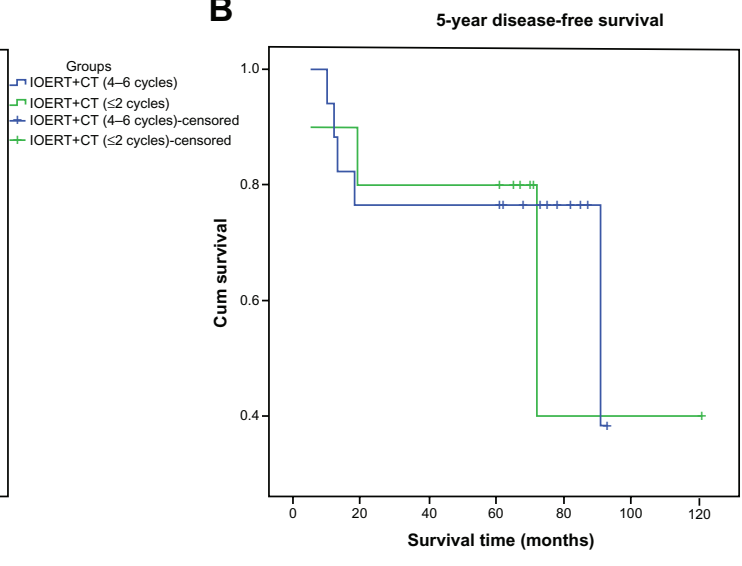

Groups
a IOERT+CT (4-6 cycles)

$\neg$ IOERT+CT ( $\leq 2$ cycles)

+ IOERT+CT $(4-6$ cycles)-censored
+ IOERT+CT $(\leq 2$ cycles)-censored
D

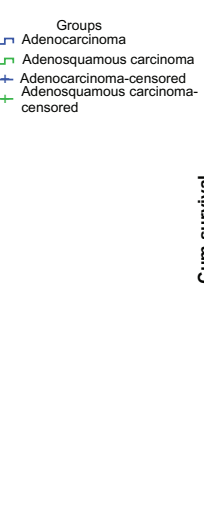

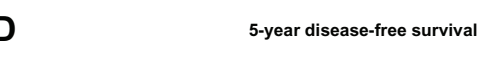

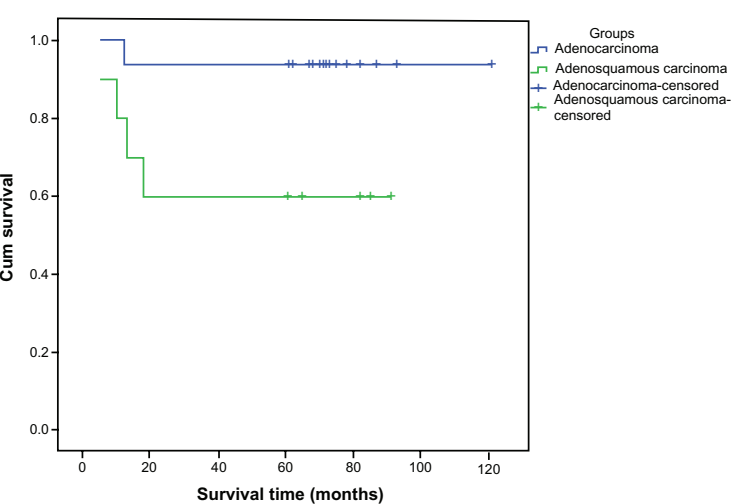

Figure I Survival analysis in advanced adenocarcinoma of the uterine cervix after IOERT.

Notes: (A) Overall survival curve stratified for EBRT + IOERT + CT (4-6 cycles) versus EBRT + IOERT + CT ( $\leq 2$ cycles) $(P>0.05)$. (B) Disease-free survival curve stratified for EBRT + IOERT + CT (4-6 cycles) versus EBRT + IOERT + CT ( $\leq 2$ cycles) $(P>0.05)$. (C) Overall survival curve stratified for adenocarcinoma versus decreased adenosquamous carcinoma $(P<0.05)$. (D) Disease-free survival curve stratified for adenocarcinoma versus decreased adenosquamous carcinoma $(P<0.05)$.

Abbreviations: CT, computed tomography; Cum, cumulative; EBRT, external beam radiation therapy; IOERT, intraoperative electron beam radiation therapy.

of 305 patients that 5 -year OS rates of stage I AC were $80 \%$, of stage II were $37 \%$, and of stage IIIb were $11 \%$. Therefore, innovative treatments to improve the survival and local control of AC are urgently needed.

In this study, we found IOERT to be both well tolerated and effective in patients with stage II AC in regard to survival benefit (the 5-year OS rate was 77.8\%) and lower number of toxicities. In addition, the findings from this study suggest that the OS in AC is higher than that in ASC. This is consistent with reports that ASC of the cervix has a poorer prognosis than AC. ${ }^{17,18}$ Although the patients with AC got survival benefit from IOERT, the 5-year OS rate and DFS rate seems still lower than that of the patients with SCC who were treated with IOERT. ${ }^{19}$ It seems to be in accord with the studies showed that AC carries a worse prognosis than SCC. ${ }^{15,16}$

IOERT also appeared to confer a disease control benefit to surgical resection, as no patient experienced disease relapse within the IOERT field, despite positive resection margins in two patients, and close margins in another six patients. Disease control was also satisfactory, with only two local failures. Thus, IOERT could potentially sterilize any microscopic residual disease in the operative bed. The aggressive

Table 2 Five-year disease-free and overall survival rates stratified by patient group

\begin{tabular}{|c|c|c|c|c|c|c|}
\hline & \multicolumn{2}{|l|}{ Treatment } & \multirow[t]{2}{*}{$P$-value } & \multicolumn{2}{|l|}{ Histologic subtype } & \multirow[t]{2}{*}{$P$-value } \\
\hline & $\begin{array}{l}\text { EBRT + IOERT + CT } \\
\text { (4-6 cycles) }\end{array}$ & $\begin{array}{l}\text { EBRT + IOERT + CT } \\
\text { ( }<2 \text { cycles })\end{array}$ & & Adenocarcinoma & $\begin{array}{l}\text { Adenosquamous } \\
\text { carcinoma }\end{array}$ & \\
\hline Overall survival & $76.4 \%(13 / 17)$ & $80 \%(8 / 10)$ & 0.832 & $87.5 \%(15 / 16)$ & $70 \%(6 / 10)$ & 0.034 \\
\hline Disease-free survival & $70.6 \%(12 / 17)$ & $70 \%(7 / / 0)$ & 0.852 & $81.3 \%(14 / 16)$ & $60 \%(5 / 10)$ & 0.031 \\
\hline
\end{tabular}

Abbreviations: CT, computed tomography; EBRT, external beam radiation therapy; IOERT, intraoperative electron beam radiation therapy. 
surgical resection of recurrent disease can result in long-term freedom from disease. However, in some cases, extensive surgery is not technically feasible, as the risk of morbidity from a resection, such as pelvic exenteration, would be too great. However, by combining surgery with IOERT, an appropriate balance of surgical aggressiveness and patient treatment-induced morbidity was achieved.

In addition, our study showed that five patients (18.5\%) had distant metastasis (including two cases of relapse para-aortic nodal metastasis, one case of liver metastasis, one case of multiple metastasis, and one case of lung metastasis), which was consistent with some studies that reported differences in patterns of disease dissemination for patients with AC histology compared to SCC of the cervix. Patients with AC had a significantly higher rate of ovarian metastasis, consistent with a number of studies that have demonstrated higher rates of distant metastasis with $\mathrm{AC}$ histology, such as ascites, abdominal carcinomatosis, and para-aortic spread. ${ }^{20}$

Some studies have reported that systemic chemotherapy and the addition of adjuvant radiotherapy plays a greater importance in the treatment of AC compared to SCC in improving survival and reducing the risk of recurrence. This may be because they harbor more micro-metastasis than $\mathrm{SCC}$, and can therefore be potentially eradicated by RT and have more to gain from the added systemic effect of extra chemotherapy courses. Additionally, further analyses of all patients receiving chemotherapy found that higher numbers of chemotherapy courses were associated with improved progression-free and overall survival. ${ }^{20}$ However, in this study, the difference in OS and DFS did not reach statistical significance between patients who had four to six courses of chemotherapy, less than four courses of chemotherapy, or no chemotherapy. Further studies about this should be anticipated.

In conclusion, IOERT may be feasible and effective as a boosting technique for advanced or recurrent AC. IOERT plus surgery may achieve high locoregional disease control and survival benefit with a low risk of toxicity, even in patients with positive resection margins. With this combined modality approach, we expect some patients with AC to experience long-term freedom from recurrence. The findings of this study confirm our clinical impressions and provide important information with which to move forward in developing better therapies for advanced $\mathrm{AC}$ of the uterine cervix.

However, the study has its own limitation. The study's results are limited by its retrospective design and the data is from a single institution. Thus, the plans for a randomized trial would be anticipated in the future.

\section{Conclusion}

IOERT may be feasible and effective as a boosting technique for advanced AC. IOERT plus surgery may achieve high locoregional disease control and survival benefit with a low risk of toxicity, even in patients with positive resection margins. With this combined modality approach, some patients with $\mathrm{AC}$ will be able to experience long-term freedom from recurrence.

\section{Acknowledgments}

This work is supported by the National Natural Science Foundation of China (No 81071838 and No 81301937), the Shaanxi Province Department of Health Key Program (No 2010A02), the International Cooperation Foundation of Shaanxi Province of China (No 2013KW-27-03), and the Foundation of the First Affiliated Hospital of Medical College of Xi' an Jiaotong University (No 2010YK3).

\section{Disclosure}

The authors declare no conflicts of interest in this work.

\section{References}

1. Wang SS, Sherman ME, Hildesheim A, Lacey JV, Devesa S. Cervical adenocarcinoma and squamous cell carcinoma incidence trends among white women and black women in the United States for 1976-2000. Cancer. 2004;100(5):1035-1044.

2. Quinn MA. Adenocarcinoma of the cervix - are there arguments for a different treatment policy? Curr Opin Obstet Gynecol. 1997;9(1): $21-24$.

3. Grisaru D, Covens A, Chapman B, et al. Does histology influence prognosis in patients with early-stage cervical carcinoma? Cancer. 2001;92(12):2999-3004.

4. Lanciano R, Calkins A, Bundy BN, et al. Randomized comparison of weekly cisplatin or protracted venous infusion of fluorouracil in combination with pelvic radiation in advanced cervix cancer: a gynecologic oncology group study. J Clin Oncol. 2005;23(33):8289-8295.

5. Monk BJ, Wang J, Im S, et al; Gynecologic Oncology Group; Southwest Oncology Group; Radiation Therapy Oncology Group. Rethinking the use of radiation and chemotherapy after radical hysterectomy: a clinical-pathologic analysis of a Gynecologic Oncology Group/ Southwest Oncology Group/Radiation Therapy Oncology Group trial. Gynecol Oncol. 2005;96(3):721-728.

6. Rotman M, Sedlis A, Piedmonte MR, et al. A phase III randomized trial of postoperative pelvic irradiation in Stage IB cervical carcinoma with poor prognostic features: follow-up of a gynecologic oncology group study. Int J Radiat Oncol Biol Phys. 2006;65(1):169-176.

7. Willett CG, Suit HD, Tepper JE, et al. Intraoperative electron beam radiation therapy for retroperitoneal soft tissue sarcoma. Cancer. 1991;68(2): 278-283.

8. Gunderson LL. Rationale and results of intraoperative radiation therapy. Cancer. 1994;74(2): 537-541.

9. Azinovic I, Martinez Monge R, Javier Aristu J, et al. Intraoperative radiotherapy electron boost followed by moderate doses of external beam radiotherapy in resected soft-tissue sarcoma of the extremities. Radiother Oncol. 2003;67(3):331-337.

10. Gao Y, Liu Z, Chen X, Luo W, Zhang L, Wang J. Intraoperative radiotherapy electron boost in advanced and recurrent epithelial ovarian carcinoma: a retrospective study. BMC Cancer. 2011;11:439. 
11. Dowdy SC, Mariani A, Cliby WA, et al. Radical pelvic resection and intraoperative radiation therapy for recurrent endometrial cancer: technique and analysis of outcomes. Gynecol Oncol. 2006;101(2):280-286.

12. Barney BM, Petersen IA, Dowdy SC, Bakkum-Gamez JN, Haddock MG. Long-term outcomes with intraoperative radiotherapy as a component of treatment for locally advanced or recurrent uterine sarcoma. Int J Radiat Oncol Biol Phys. 2012;83(1):191-197.

13. Tran PT, Su Z, Hara W, Husain A, Teng N, Kapp DS. Long-term survivors using intraoperative radiotherapy for recurrent gynecologic malignancies. Int J Radiat Oncol Biol Phys. 2007;69(2):504-511.

14. National Cancer Institute (March 31,2003). Cancer Therapy Evluation Program. Common Terminology Criteria for Adverse Events. Version3.0. Available from http://cteo.cancer.gov. Accessed July 20, 2013.

15. Davy ML, Dodd TJ, Luke CG, Roder DM. Cervical cancer: effect of glandular cell type on prognosis, treatment, and survival. Obstet Gynecol. 2003;101(1):38-45.

16. Baalbergen A, Ewing-Graham PC, Hop WC, Struijk P, Helmerhorst TJ. Prognostic factors in adenocarcinoma of the uterine cervix. Gynecol Oncol. 2004;92(1):262-267.
17. Farley JH, Hickey KW, Carlson JW, Rose GS, Kost ER, Harrison TA. Adenosquamous histology predicts a poor outcome for patients with advanced-stage, but not early-stage, cervical carcinoma. Cancer. 2003;97(9):2196-2202.

18. Wang SS, Sherman ME, Silverberg SG, et al. Pathological characteristics of cervical adenocarcinoma in a multi-center US-based study. Gynecol Oncol. 2006;103(2):541-546.

19. Liu Z, Gao Y, Soong YL, et al. Intraoperative electron beam radiotherapy for primary treatment of stage IIB cervical cancer: a retrospective study. $J$ Int Med Res. 2012;40(6):2346-2354.

20. Gien LT, Beauchemin MC, Thomas G. Adenocarcinoma: a unique cervical cancer. Gynecol Oncol. 2010;116(1):140-146.

21. Peters WA, Liu PY, Barrett RJ, et al. Concurrent chemotherapy and pelvic radiation therapy compared with pelvic radiation therapy alone as adjuvant therapy after radical surgery in high-risk early-stage cancer of the cervix. J Clin Oncol. 2000;18(8):1606-1613.
OncoTargets and Therapy

\section{Publish your work in this journal}

OncoTargets and Therapy is an international, peer-reviewed, open access journal focusing on the pathological basis of all cancers, potential targets for therapy and treatment protocols employed to improve the management of cancer patients. The journal also focuses on the impact of management programs and new therapeutic agents and protocols on

\section{Dovepress}

patient perspectives such as quality of life, adherence and satisfaction The manuscript management system is completely online and includes a very quick and fair peer-review system, which is all easy to use. Visit http://www.dovepress.com/testimonials.php to read real quotes from published authors. 